\title{
Aspectos del lenguaje económico español y alemán: cuantificadores $y$ expresiones de cantidad en contraste
}

\author{
Alice Stender \\ Universidad Pablo de Olavide \\ aste@upo.es
}

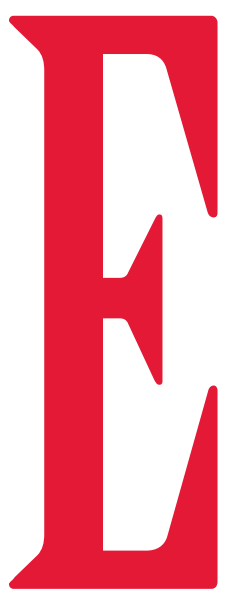

l uso de cifras es inherente a la naturaleza de los textos económicos, pues una de las funciones básicas de la economía es la de contar o cuantificar elementos -o recursos- que el ser humano, desde el principio de los tiempos, ha necesitado para sobrevivir. Hoy día los textos económicos están plagados de numerales que modifican monedas, cantidades de capital, tipos de interés, porcentajes, etcétera.

\section{Introducción}

No obstante, las cifras son solo una pequeña parte de las expresiones cuantitativas, y no podemos olvidarnos de otras expresiones cuantificadoras que son características del discurso económico, tales como los adverbios que expresan cantidad (apenas, bastante, kaum, genügend...) o adjetivos como fuerte, escaso, stark, knapp, que, como veremos más adelante, también contribuyen a la cuantificación. Comparativos, superlativos, con o sin modificadores (como superior a, más, tanto como, besser als, mehr, so viel wie), y sustantivos como repunte o Rekordhoch, completan un complejo repertorio de elementos léxicos que expresan cuantificación en las lenguas española y alemana. Por este motivo, no hablamos de numerales sino de cuantificadores o expresiones cuantitativas.

Nuestro objetivo en este estudio es el de presentar algunas de las diferencias más destacables entre el alemán y el castellano, observar y contrastar los cuantificadores presentes en los textos económicos periodísticos de ambos países y establecer cuáles son los rasgos comunes y cuáles los diferenciadores en el lenguaje económico de la prensa especializada. Nuestro propósito es el de conocer la lengua en su uso real y que estos conocimientos puedan servir, entre otros, para la enseñanza del lenguaje económico, así como para el ejercicio de la traducción especializada en las lenguas de nuestro interés.

Para ello, nos servimos de un corpus comparable denominado CrisCorp (Medina Reguera y Stender 2012, 2013; Stender 2016) que incluye textos de la prensa económica especializada en torno a la crisis económica de $2007^{(1)}$. Para lograr nuestros objetivos expondremos, en primer lugar, qué es la cuantificación y qué tipos de expresiones cuantitativas existen en ambas lenguas según las referencias bibliográficas más destacadas y, en segundo lugar, observaremos algunos de estos cuantificadores en contraste basándonos, en la medida de lo posible, en los resultados que arroja nuestro corpus textual.

\section{Los estudios sobre la cuantificación del español y el alemán}

Labrador (2005: 45) se basa en diferentes definiciones de 'cantidad' para concluir que "la cantidad siempre es relativa; una misma cantidad puede ser enorme o minúscula, según aquello que se compare o el fin para que sirva”, y afirmar que existen distintos tipos de medida que se emplean de manera global con el objetivo de "cuantificar objetos y fenómenos físicos" y que, generalmente, es la mensurabilidad y la calidad desde un punto de vista matemático y filosófico lo que se relaciona con la cantidad. La relatividad a la que hace 


\section{Abstract:}

The presence of numbers and ciphers is representative for texts on economics as one of the aims of economics is to quantify different elements, such as currencies, amounts of capital or interest rates. However, this is just one way to express quantity, because there are also adjectives such as stark, fuerte ('strong') or knapp, escaso ('scarce') or adverbs such as kaum, apenas ('hardly') or genügend, suficiente ('enough'). Also, nouns such as mogollón ('lots of') or Rekordhoch ('record high') play a fundamental role in the language of economics, not to mention different ways of gradation such as besonders, especialmente ('particularly"). Our aim in this study is to present some of the most significant differences and similarities between the Spanish and German languages of economics. The quantifiers, which are present in texts of different economics journals, will be analysed and compared in order to discover the related characteristics of these two languages. The knowledge of the respective features of both languages can be of interest for specialist language teaching, with emphasis on texts on economics, and for technical translators. To achieve this goal we have researched relevant authors in both languages, and examined a corpus of 500 texts in Spanish and German dealing with the international economic crisis, between 2007 and 2015, in search of quantifiers.

Keywords: Quantifiers, language of economics, corpus, German, Spanish.

\section{Zusammenfassung}

Die Präsenz von Ziffern und Zahlen ist aus ökonomischen Texten nicht wegzudenken, denn eine Funktion der Wirtschaft ist es, verschiedene Elemente zu quantifizieren. Sie werden dazu benutzt, um unter anderem Währungen, Kapitalmengen, Zinssätze oder Prozente zu beschreiben. Allerdings ist die Anwendung von Zahlen nur eine von vielen Möglichkeiten, Menge auszudrücken, denn in der Wirtschaftssprache werden auch Adjektive wie stark, knapp oder Adverbien wie kaum oder genügend angewendet. Zudem spielen Substantive wie Rekordhoch oder Dualia (beide) neben Grad- oder Fokuspartikeln wie ausgerechnet oder besonders eine wichtige Rolle bei der Quantifizierung. Unser Ziel ist es, in dieser Studie einige der signifikantesten Unterschiede und Gemeinsamkeiten zwischen der spanischen und der deutschen Wirtschaftssprache zu präsentieren. Die Quantoren, die in Texten verschiedener Wirtschaftszeitschriften Verwendung finden, werden identifiziert und miteinander verglichen, um die diesbezüglichen Eigenschaften dieser beiden Sprachen zu erforschen. Die Kenntnisse über die jeweiligen Merkmale beider Sprachen sollen in den Unterricht für Fachsprachen, insbesondere mit wirtschaftlichem Schwerpunkt, einfließen und bei Fachübersetzungen im Wirtschaftsbereich helfen. Mit diesem Ziel haben wir die Literatur zum Thema in unserem Sprachenpaar recherchiert und ein Korpus aus 500 Texten, mit Thematik der Wirtschaftskrise von 2007 bis 2015, auf Quantoren untersucht.

Schlüsselwörter: Quantoren, Wirtschaftssprache, Korpus, Deutsch, Spanisch. alusión Labrador también se hace extensible a la relación existente entre receptor y emisor de un enunciado, ya que la forma en la que el receptor interpreta una cantidad depende en ocasiones de su propio conocimiento del mundo.

La autora señala también la necesidad de diferenciar entre la función cuantificadora (¿qué cuantifica?) y el elemento cuantificador (i.e. una cifra o un adjetivo gradativo, etc.) ya que un elemento puede cumplir con diferentes acometidos.

Existe una clara dificultad a la hora de establecer qué es un cuantificador, sobre todo dada la gran variedad de categorías gramaticales en las que éstos se engloban. Esta falta de consenso es común a nuestro par de lenguas (Sánchez 1999, Leonetti 2007, Delbecque y Verveckken 2012, Lamíquiz 1991 para el español y Wiese 1996, Vater 1984 o Bußmann 2002 para el alemán). Como afirma Leonetti (2007: 11) "las palabras que hoy se denominan cuantificadores son, para los gramáticos clásicos, adjetivos, pronombres o adverbios" y según él, la dificultad radica sobre todo en que vocablos como demasiado, menos, mucho o tanto pertenecen a distintas clases de palabras y no se dejan englobar en una única categoría. Él distingue entre dos grandes clases de cuantificadores: los adnominales y los adverbiales. En la primera categoría se catalogan los pronombres o adjetivos, que, en la gramática moderna se incluyen dentro de los determinantes. Ejemplos: cualquier, cuatro, alguno, tanto, menos. Este grupo comprende también diferentes clases, los cuantificadores universales (fuertes) por un lado, y los cuantificadores existenciales (débiles) por el otro y a los que también se refieren Gutiérrez Rodríguez (2008) y Verveckken (2015) como definidos e indefinidos respectivamente. Los cuantificadores universales, como todo(s), ambos ${ }^{(2)} \mathrm{o}$ cada indican la totalidad de los miembros del conjunto sobre el que se cuantifica (lo que significa que son definidos).

En cuanto a los cuantificadores existenciales, estos son los 
que incluyen a los determinantes numerales (cardinales) y a la mayoría de los indefinidos (algún, mucho, varios, ningún, bastantes), cuantificadores comparativos (más, menos, tanto) y los cuantificadores exclamativos e interrogativos y relativos (qué, cuánto, cuál, cuanto).

Kirchner (2000) hace un resumen de las gramáticas españolas y determina que a los cuantificadores corresponden palabras como adjetivos o pronombres, así como el articulo determinado, numerales y construcciones que, de alguna manera, definen cantidades (esto es, la pluralidad indefinida [millones] o definida [dos millones de euros]). Sánchez (1999) habla de los cuantificadores propios, que denotan cantidad en su forma explícita, y los focales o presuposicionales (en alemán Fokuspartikel o Gradpartikel), cuyo contenido cuantitativo es implícito, puesto que no se define una cantidad concreta, sino que se interpreta una realidad en términos de cuantificación (i.e. también).

Por otro lado, si bien una expresión cuantitativa, como fuerte retroceso, no es un cuantificador en el sentido estricto, sí es capaz de cuantificar, es decir, que el adjetivo fuerte matiza el concepto de retroceso que se puede mover entre los dos extremos de una escala y el mismo sustantivo indica una gradación. Este último caso muestra que una gradación cuantitativa también se puede obtener mediante el uso de afijos (sufijos y prefijos). En estas nuevas palabras el afijo modifica al lexema de tal manera que éste ahora presenta características que indican cantidad de un determinado grado (macroeconomía frente a microeconomía) mayor o menor, además de mostrar imprecisión ya que no reflejan cantidades exactas.

Meilán (1998: 64) hace referencia a los sustantivos en función de complemento circunstancial de cantidad que en su acepción semántica se emplean para indicar un grado de cantidad máxima. Él da como ejemplo, entre otras, las palabras mogollón, horrores o la tira. Sánchez (1999: 1050) opina que, a pesar de que no se trata de nombres partitivos intrínsecos debido a su carácter descriptivo, sí "denotan una cantidad aproximada" y Labrador (2005: 65) habla de cuantificación lexemática para referirse al "hecho de que muchos lexemas, en su propia configuración sémica poseen algún rasgo que alude a cierta cantidad, elevada o pequeña” para lo que no solamente menciona a los sustantivos, sino también determinados verbos, adjetivos, etc.

Si bien podemos observar que algunos lingüistas establecen diferentes clasificaciones, todos están de acuerdo en que se trata de un tema bastante confuso por lo que Lamíquiz incluso afirma en 1991 que "el tema de la cuantificación lingüística ofrece el conjunto más complejo de problemas en toda la descripción de la lengua" y Leonetti (2007: 10) alega que existe "un complejo panorama de la semántica de la cuantificación".

Labrador (2005) distingue de manera global entre tres tipos de cuantificación: la precisa, la imprecisa y la comparativa, que, a su vez, se dividen en otras tantas subclases que resume en la siguiente tabla:

\begin{tabular}{|l|l|l|l|l|}
\hline Precisa & Imprecisa & \multicolumn{2}{|c|}{ Comparativa } \\
\hline Numeral & -cardinal & Indefinida & Comparativa & -superioridad \\
\cline { 2 - 3 } & -ordinal & Partitiva & & -igualdad \\
\cline { 2 - 3 } & -fraccionaria & Colectiva & & -inferioridad \\
\cline { 2 - 3 } & -multiplicativa & Iterativa & Superlativa & -superioridad \\
\cline { 2 - 3 } & -distributiva & Afijativa & & -inferioridad \\
\cline { 2 - 3 } & -colectiva & Lexemática & & \\
\cline { 2 - 3 } & -dual & Aproximativa & \\
\hline Unidades de medida & Entonativa & \\
\hline \multicolumn{2}{|l}{} & $\begin{array}{l}\text { Focal o } \\
\text { presuposicional }\end{array}$ & & \\
& & & \\
\end{tabular}

Tabla 1: Tipología de la cuntificación según Labrador (2005).

En la interpretación de esta tabla cabe seguir su reflexión según la cual para algunos autores, por ejemplo Alarcos (1994), solamente los números cardinales pertenecen a la cuantificación numeral - por un lado en su expresión escrita como cifra y por el otro en su forma desarrollada- mientras que para otros hay más clases que pueden pertenecer a los numerales ya que de una forma u otra tienen "en muchas ocasiones una función cuantificadora" (Labrador 2005: 57).

Martínez (2007: 170) afirma: "Solo los números cardinales expresan una cantidad exacta; los demás realizan una cuantificación indeterminada, y constituyen una gradación que va desde la totalidad de todo hasta la ausencia de cantidad de nada y ninguno. [...] prácticamente todas las referencias a la realidad son cuantificables".

Si solo los números cardinales son los que indican cantidades exactas, para las mediciones inexactas hay que tener en cuenta el concepto de los denominados cuantificadores imprecisos (o "vagos" (3) como los designan algunos autores, entre ellos Zamparelli 2000) como, por ejemplo, algunos o muchos, einige, viele etc. Wang y Piao (2007) hacen constar que la vaguedad en lingüística se refiere generalmente, pero no de manera exclusiva, a "the indeterminacy of the referencial boundary of words". Ellos resumen que, en las distintas investigaciones, se mencionan generalmente dos tipos de imprecisión: la lingüística, es decir las propias palabras que contienen una gradación en $\mathbf{s i ́}^{\prime(4)}$, y la pragmalingüística que se refiere a "certain expressions 
that are considered as vague". Se consideran expresiones imprecisas porque la interpretación del mensaje depende del punto de vista del receptor. El gradativo proporcional mucho, por ejemplo, no indica una medida clara que dice cuánto es mucho ya que para el magnate Bill Gates un millón de dólares probablemente no es mucho dinero mientras que sí lo es para la mayoría.

Este tipo de cuantificador es el que mide a las sustancias incontables y Labrador (2005) sitúa a los cuantificadores indefinidos en una escala de gradación donde los cuantificadores negativos y los proporcionales de inferioridad se ubican en un extremo y los universales y proporcionales de superioridad en el otro, mientras que los cuantificadores existenciales y los relacionales se mueven en el medio de esta escala.

En lo que se refiere a la cuantificación imprecisa partitiva, ésta se compone generalmente de sustantivos que indican una cantidad grande o pequeña y que muchas veces se encuentran acompañados por adjetivos que enfatizan la cantidad, así como también pueden hacer referencia a la forma en la que se manifiestan.

La cuantificación colectiva ${ }^{(5)}$ y la partitiva, dentro de la clasificación imprecisa, tienen muchas nociones en común, ya que las dos se refieren a partes de un conjunto, si bien los partitivos "suelen actualizar sustantivos incontables y los colectivos sustantivos contables" (ibíd. 2005: 63).

La cuantificación imprecisa, y a pesar de la opinión quizás generalizada de que los economistas deberían hablar en números concretos, no se expresa solamente con este tipo de cuantificadores, sino existe una tendencia clara en los textos periodísticos de nuestro corpus de compendiar grandes cifras en totales como, por ejemplo, cuatro millones, en vez de $3.987,000$, lo que Labrador también ubica dentro de la cuantificación aproximativa.

Si la clasificación en los estudios gramaticales o lingüísticos para el español es, cómo poco, difícil y variada, en alemán no resulta muy diferente y los problemas se basan también en la divergencia de todas las clases de palabras que semánticamente tienen la capacidad de expresar cuantificación. Bußmann (2002: 547) define los cuantificadores (Quantor, Quantifikator o Zahlwort) como términos para la descripción de determinantes que sirven para la cuantificación y que engloban a determinados adjetivos, pronombres y a los numerales. Vater (1984) declara que los cuantificadores son "sprachliche Ausdrücke, die eine Quantität bezeichnen" y el mismo Vater (1996: 195) afirma que "ein weiteres Indiz für die syntaktische
Verschiedenheit von Determinantien und Quantoren ist die Tatsache, dass nur Quantoren distanziert werden können“.

Gottschall (2008: 52) escribe que "Ausdrücke, die sich nicht auf ein bestimmtes Individuum beziehen, sondern die eine Aussage darüber erlauben, auf wie viele Individuen ein Prädikat zutrifft, heißen Quantifikatoren oder Quantoren “ $y$ Wiese (2001: 2) cita a Hirt (1927: 306f) cuando este afirmó a final de los años veinte que "die Zahlwörter bilden eine eigene Kategorie, die man nicht recht einordnen kann". Heibig y Buscha (1996: 21) por su parte opinan que „Numeralia und Pronomina stellen keine Wortklassen im syntaktischen Sinne dar, sondern füllen verschiedene syntaktische Funktionen aus: Sie fungieren teils als Substantive, teils als Adjektive, teils als Artikelwörter".

Ballweg (2003: 13) también habla de distintas categorías entre las que destacan los determinantes (der, alle), adjetivos (viel, wenig), medidas (ein Liter, ein Pfund) o expresiones de contenido (ein Glas). Podemos afirmar entonces, que en ambas lenguas de nuestro interés convive una gran variedad de clases de palabras que sirven para la cuantificación y que en algunos casos no existe un consenso entre los diversos autores a la hora de clasificar dichas variedades.

Si bien, generalmente, también en alemán, y según el Handbuch der deutschen Grammatik (2003: 256), se distingue entre

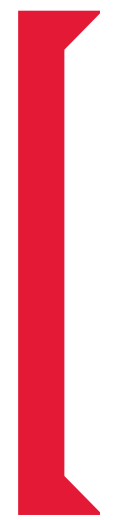

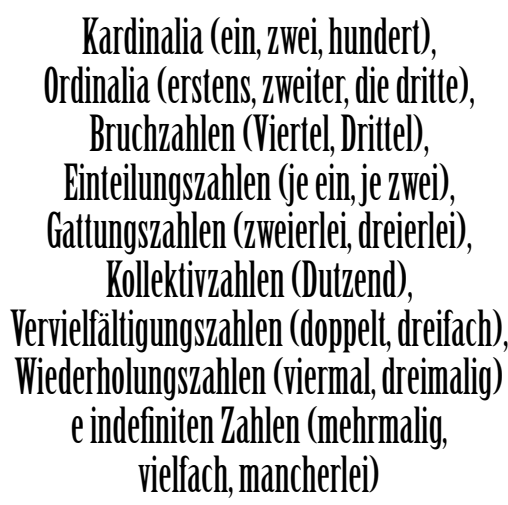

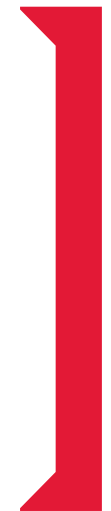

En alemán los numerales no se comportan de manera uniforme con respecto a la morfología o la sintaxis de la gramática general ya que algunos se declinan conforme a las normas gramaticales y otros no.

Otra forma de expresar cantidad es, asimismo, la creación de plurales o de singulares, así como las expresiones que solamente se emplean en plural (pluraliatantum) o en singular (singulariatantum), si bien Eschenbach (2013: 51) advierte que en los lenguajes especializados existe la tendencia de crear plurales debido a los nuevos referentes 
(Sand/Sande) a pesar de que la mayoría de estos singulares es incontable y carece, por lo tanto, de una forma en plural. El autor distingue entre características cuantitativas y estructurales y concluye que las dimensiones dependen menos de la expresión lingüística que del conocimiento conceptual:

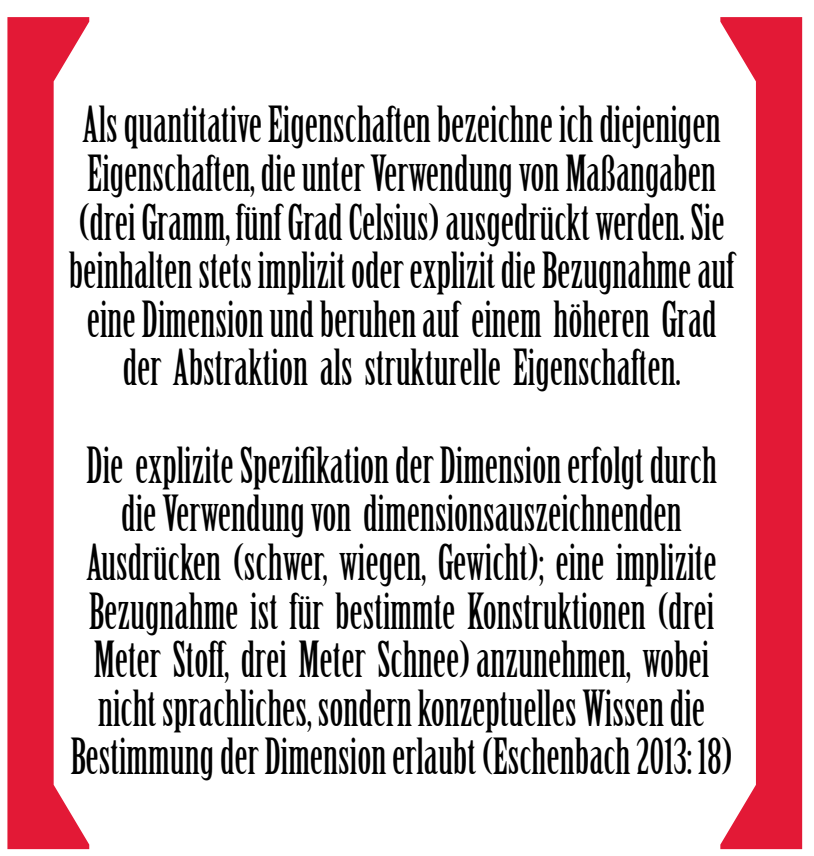

Löbner(1985:79) afirma por su partequela cuantificación es un fenómeno muy extendido en las lenguas naturales que se sirven de expresiones de las más diversas categorías y, además, asevera que „[z]um einen gibt es zwischen kein und jeder eine grosse Skala von Abstufungen, deren Elemente innerhalb der Determinatoren Subkategorien bilden" (Löbner ibíd: 95), mientras que Gvozdanović (2006) se centra en los adverbios y menciona al respecto los adverbios aspectuales con características cuantificadoras (i.e. immer).

Un elemento que sirve para la cuantificación positiva son
Autores como Hentschel y Weidt (1989), König (1981) y Altmann (1978) prefieren hablar de Fokuspartikeln. Heibig y Buscha (1996: 478) también los denominan Intensifikatoren y Brauße (2000) afirma que las "Fokuspartikeln sind die Partikeln, die gemeinsam mit ihrer Fokuskonstituente im Satz wandern können (und für die gleichbedeutend heute ebenfalls die Bezeichnung "Gradpartikeln" eingeführt ist".

Estos elementos, aunque también reciben el nombre de Gradpartikeln -lo que de por sí implica una función de gradación- destacan el elemento más importante de la oración (auch, ausgerechnet, besonders, insbesondere, nur, sogar, wenigstens...), pero no todas tienen la función de graduar. Weinrich (2005: 510), por ejemplo, prefiere hablar de GradAdverbien que, a su vez, distingue en diferentes subclases.

Por supuesto, también se puede cuantificar mediante la composición del lexema con determinados elementos para expresar el alto grado de algo, como, por ejemplo: hellwach o bitterböse, por mencionar los ejemplos que usa Eschenbach (2013). O, igual que en español, por afijación.

Wiese (2004: 5) explica los campos de actuación de los distintos tipos de cuantificadores en alemán. Los números cardinales ayudan en los contextos en los que hay que contar un determinado número de elementos (3 Bleistifte= 3 lápices), así como cuando se trata de medir cantidades. Los números ordinales "identifizieren Zahlen innerhalb einer Sequenz" (der dritte Läufer=el tercer corredor), mientras que los cuantificadores nominales hacen referencia a objetos dentro de un conjunto, como la numeración de líneas de autobús (Bus Nr. 3=autobús n. ${ }^{\circ}$ ).

Llegados a este punto $y$ tras revisar la literatura pertinente, afirmamos que existe un panorama un tanto confuso en ambas lenguas. Para tratar de arrojar algo de luz ofrecemos la siguiente tabla basada en la clasificación de los cuantificadores propios de Sánchez (1999) a la que añadimos ejemplos en alemán y en español procedentes de nuestro corpus.

las Intensivpartikeln como ganz, höchst, recht, sehr, überaus, ziemlich, zutiefst, etc. a las que se recurre para, como bien indica la palabra, aumentar o disminuir la intensidad de un enunciado semánticamente graduable $\mathrm{y}$ para modificar el significado de un adjetivo de manera que se puede mover a lo largo de una escala (sehr viel, viel, recht viel, noch viel, ziemlich wenig, wenig, ganz wenig).

\begin{tabular}{|c|c|c|c|c|}
\hline \multirow{2}{*}{\multicolumn{2}{|c|}{ CUANTIFICADORES PROPIOS }} & \multirow{2}{*}{ SÁNCHEZ } & \multicolumn{2}{|c|}{ CRISCORP } \\
\hline & & & ESPAÑOL & ALEMÁN \\
\hline \multirow{5}{*}{ Numerales } & cardinales & uno, dos, mil,... & menos de tres horas & von vier Prozent \\
\hline & ordinales & primero, décimo, ... & el primero en pedir ayudas & $\begin{array}{l}\text { das erste Mal, am zweiten Tag in } \\
\text { Folge }\end{array}$ \\
\hline & partitivos & mitad, tercio, $\ldots$ & un cuarto de punto & Die Hälfte ihres Marktwerts, \\
\hline & multiplicativos & doble, triple,... & la triple A & $\begin{array}{l}\text { Zweckgesellschaften sind gleich } \\
\text { doppelt }[\ldots] \text { betroffen, }\end{array}$ \\
\hline & distributivos & Sendos & $\begin{array}{l}\text { No hay ejemplos de sendos en } \\
\text { CrisCorp }\end{array}$ & $\begin{array}{l}\text { mit ihren jeweiligen internationalen } \\
\text { Partnerbehörden }\end{array}$ \\
\hline \multirow{5}{*}{ Indefinidos } & universales & $\begin{array}{l}\text { todo, cada, cada uno, ambos, } \\
\text { cualquiera }\end{array}$ & $\begin{array}{l}\text { para todo el mundo, cinco de cada } \\
\text { lado, un 2,15\% cada uno, ambas } \\
\text { cámaras, cualquier barrera, }\end{array}$ & $\begin{array}{l}\text { alle ihm zur Verfügung stehenden } \\
\text { Mittel, beide Kammern, }\end{array}$ \\
\hline & no universales & algo, alguien, uno, alguno & $\begin{array}{l}\text { como algo puntual, alguien (no hay), } \\
\text { uno de los peores, algunos } \\
\text { inversores }\end{array}$ & $\begin{array}{l}\text { legten vorbörslich etwas zu, jeder } \\
\text { wartet irgendwie darauf, }\end{array}$ \\
\hline & afirmativos & $\begin{array}{l}\text { varios, pocos, mucho, bastante, } \\
\text { demasiado, nada }\end{array}$ & $\begin{array}{l}\text { varios analistas, pocos amigos, } \\
\text { bastante negativos, han alentado } \\
\text { demasiado el ánimo, han sido nada } \\
\text { positivas }\end{array}$ & $\begin{array}{l}\text { Notenbanken mehrerer Länder, } \\
\text { wenig Vertrauen, so viel Vermögen, } \\
\text { zu viel Regulierung, keine Jobs mehr }\end{array}$ \\
\hline & negativos & nadie, ninguno, & $\begin{array}{l}\text { nadie quiere volver al comunismo, } \\
\text { no quiso señalar a ninguno, }\end{array}$ & $\begin{array}{l}\text { Thain werde niemand mehr glauben, } \\
\text { so recht weiß am frühen Morgen noch } \\
\text { keiner }\end{array}$ \\
\hline & comparativos & más, menos, tanto & $\begin{array}{l}\text { más de lo que tenía previsto, quedan } \\
\text { menos de tres, tanto común como } \\
\text { preferente }\end{array}$ & $\begin{array}{l}\text { auf mehr als } 400 \text { Mrd. Dollar, } \\
\text { weniger als zehn Mill. Euro, ebenso } \\
\text { wie die Deutsche Bank }\end{array}$ \\
\hline
\end{tabular}




\begin{tabular}{|c|c|c|c|c|}
\hline \multirow{2}{*}{\multicolumn{2}{|c|}{ CUANTIFICADORES PROPIOS }} & \multirow{2}{*}{ SÁNCHEZ } & \multicolumn{2}{|c|}{ CRISCORP } \\
\hline & & & ESPAÑOL & ALEMÁN \\
\hline Gradativos & proporcionales & $\begin{array}{l}\text { algo, (un) poco, mucho, bastante, } \\
\text { demasiado, todo, nada }\end{array}$ & $\begin{array}{l}\text { algo puntual, por poco más de, } \\
\text { mucho recelo, bastante negativos, } \\
\text { han pesado demasiado en el parqué, } \\
\text { a todo tipo de bancos, ha resultado } \\
\text { nada positivo }\end{array}$ & $\begin{array}{l}\text { eine gewisse Beruhigung am Markt, } \\
\text { ausreichend } \text { Kapital, schlimm } \\
\text { genug, } \text { zu viel } \text { Regulierung, sie } \\
\text { werde alles tun, daran habe sich } \\
\text { nichts geändert, }\end{array}$ \\
\hline \multicolumn{5}{|c|}{ CUANTIFICADORES PRESUPOSICIONALES O FOCALES } \\
\hline Incluyentes & & $\begin{array}{l}\text { también, incluso, hasta, tampoco, ni } \\
\text { siquiera }\end{array}$ & $\begin{array}{l}\text { se extiende también a la temporada, } \\
\text { incluso de mañana, desde el BCE } \\
\text { hasta la FED, tampoco ha resultado } \\
\text { nada positivo, ni siquiera (no hay) }\end{array}$ & $\mid \begin{array}{l}\text { auch die Börsen in Europa, erhöhte } \\
\text { seine Verlusterwartung sogar um } 51 \\
\text { Prozent, auch sei ein Überschwappen } \\
\text { nach Europa nicht } \text { auszuschließen, es } \\
\text { sei derzeit noch gar nicht } \text { abzusehen }\end{array}$ \\
\hline Excluyentes & & sólo, al menos, apenas & $\begin{array}{l}\text { sólo siete suspendieron, al menos las } \\
\text { últimas cuentas, en apenas dos días }\end{array}$ & $\begin{array}{l}\text { nur } 4200 \text { Jobs gestrichen, wie seit } \\
\text { mindestens einem Jahr nicht mehr, } \\
\text { Staatsanleihen kaum verändert }\end{array}$ \\
\hline
\end{tabular}

del 20 al 99 o -ig (30 al 39) y en los centenares -hundert ${ }^{(6)}$ mientras que la terminación de los millares es -tausend.

En alemán, contrariamente al español, no se emplean preposiciones cuando tratamos de cantidades concretas (B_22: recibirá una inyección de 5.000 millones de dólares frente a A_22: musste Morgan 7,8 Mrd. Dollar abschreiben) ${ }^{(7)}$, pero la diferencia quizás más destacable entre el alemán y el español en lo que se refiere

Tabla 2: Cuantificadores según la clasificación de Sánchez (1999) para el español.

\section{Contraste y diferencias de uso entre el alemán y el español}

Hemos adoptado la clasificación de Sánchez (1999) para dividir los cuantificadores en numerales y sus subclases cardinales (dos, mil, zwei, tausend), ordinales (primero, décimo, erster, zehnter), partitivos o fraccionarios (cuarto, mitad, tercio, Hälfte, Viertel, Drittel), multiplicativos (doble, triple, doppelt, dreifach) y distributivos (sendos, jeweilige). Además añadimos, siguiendo la clasificación de Labrador (2005), los colectivos (par, trio, Paar, Trio) y los cuantificadores duales (ambos, beide) para nuestro análisis.

A la hora de contrastar las dos lenguas atendiendo a las clasificaciones anteriores, en primer lugar, quisiéramos llevar la atención a los numerales (Numeralia o Zahlwörter en alemán) dada su importancia en los textos económicos y su significación unívoca, si bien también aquí existe una "problematische Wortartzugehörigkeit [...] (semantisch-konzpetuelle und phonolgisch-lexikalische Zusammengehörigkeit versus morphosyntaktische Heterogenität" (Wiese 2001: 12). Los cuantificadores numerales pertenecen a la clase de los cuantificadores precisos ya que el número indica una cantidad concreta. En ambas lenguas estos elementos funcionan únicamente como nombres. En cuanto a la creación de los numerales en alemán se utilizan las terminaciones -zig para los números a los números cardinales, se centra en la traducción de determinadas cantidades de millones. Así, el equivalente a mil millones es Milliarde, mil billones equivalen a eine Billiarde y mil trillones a eine Trilliarde (Castell 2002: 365). También Pérez Berenguel (2003: 622) se refiere a esta diferencia y afirma que la RAE ha acordado la "acuñación del término milliardo, no sabemos aún con qué grado de éxito".

Para contrastar el uso que cada lengua hace de las clases de cuantificadores arriba mencionados, hemos llevado a cabo un recuento semiautomático de los cuantificadores presentes en nuestro corpus. En el caso de esta cuantificación cardinal destaca, sobre todo, el uso de abreviaturas en alemán y la total ausencia de equivalentes abreviados en español como se puede ver en la siguiente tabla:

\begin{tabular}{|l|c|l|c|}
\hline ALEMÁN & FRECUENCIA & ESPAÑOL & FRECUENCIA \\
\hline Mill. & 29 & Billón & 6 \\
\hline Mio. & 28 & Billones & 25 \\
\hline Millionen, Million & $88+1$ & Millones & 808 \\
\hline Milliardär & 8 & Millón & 1 \\
\hline Milliarde & 13 & Multimillonario & 11 \\
\hline Milliarden & 361 & & \\
\hline Mrd. & 235 & & \\
\hline $\begin{array}{l}\text { Diferentes compuestos con } \\
\text { Milliarden }\end{array}$ & 77 & & \\
\hline Billion & 7 & & \\
\hline Bill. & 4 & & \\
\hline Billionen TOTAL & $\mathbf{8 6 9}$ & & \\
\hline & & & TOTAL \\
\hline
\end{tabular}

Tabla 3: Sustantivos de significación numeral y su composición. 
La tabla superior demuestra que el alemán emplea muchas más formas compuestas que el español, pero a pesar de esta divergencia, en total hay mucha coincidencia en el número de sustantivos de significación numeral, ya que el número hallado en ambos subcorpus es prácticamente el mismo (869 del subcorpus alemán frente a 851 en el subcorpus español).

Los números ordinales se distinguen por una característica ortotipográfica, pues si en alemán se escriben cifras, éstas han de ir seguidas por un punto como en el siguiente ejemplo: 4. = viert - = cuarto. Destacable es también la diferencia de escritura de las indicaciones temporales referidas a los siglos, pues mientras que en español la norma más difundida es la de escribir en números romanos (siglo XXI), en alemán se emplean los números arábigos ordinales (21. Jahrhundert).

La clasificación continúa con los números partitivos (Bruchzahlen): mitad o medio, cuarto, quinceavo, Hälfte ${ }^{(9)}$, Viertel(10), viertel, fünfzehntel, etc.; y los iterativos (dos veces, zweimal) y multiplicativos (triple, dreifach). Las iteraciones terminan en alemán en -malig y las multiplicaciones en -fach ${ }^{(11)}$. Castell (2002), en su gramática contrastiva, hace hincapié en que el contraste entre la multiplicación y la iteración es, sobre todo, temporal. Igual que los números cardinales, también los partitivos españoles necesitan de la preposición de, contrario al alemán que prescinde de tal elemento.

Martínez (2007) recuerda que las palabras millón, billón, etcétera, son palabras especialmente adecuadas para la cuantificación de otros sustantivos, entendemos que además cientos de (2) y miles de (7), así como Hunderte (3) y Tausende (3) sirven para este propósito. También en alemán se emplean estas palabras con función partitiva, tanto con la preposición von, como sin ella. No es muy frecuente el uso de los millares en el subcorpus español (solamente en forma de cifras), pues aparecen tan solo ocho veces, como tampoco lo es en el subcorpus alemán (10) y en las dos lenguas solamente se emplea para indicar magnitud imprecisa (A_153: Leidtragende sind Zehntausende Kleinsparer, B_30: Los ahorros de miles de familias).

$\mathrm{Si}$ bien existen generalmente correspondencias de los números partitivos formados a partir de los números ordinales (tercio, veinteavo, drittel, zwanzigstel), no es así en el caso de los números partitivos como decena, quincena, etcétera por lo que es usual emplear en una traducción los números cardinales propios o incluso la expresión coloquial $z i^{(13)}$, con el significado de mucho (Ejemplos: quincena: zwei Wochen, vierzehn Tage. Hubo decenas de despidos: Es gab zig/viele/eine große Anzahl Entlassungen).

Otra diferencia entre el castellano y el alemán es el uso de los colectivos par, paar y Paar. Aunque no aparecen en el corpus, se merecen una mención especial ya que en alemán existe un sustantivo cuantificador preciso (Paar) cuya finalidad es, según Weinrich (2005), la de designar a parejas de humanos o de animales o prendas de vestir con dos partes simétricas (ein Paar Schuhe), mientras que en español depende del contexto, pues par puede pertenecer tanto a la cuantificación precisa (un par de zapatos) como a la imprecisa (un par de años, un par de veces), en cuyo caso se trataría del Schätz-Artikel (Weinrich 2005) paar (ein paar Jahre, ein paarmal).

Los cuantificadores universales son imprecisos, ya que indican una parte entera, la totalidad de un conjunto, de algo, pero no revelan de cuántas personas o cosas se trata. El equivalente alemán del cuantificador distributivo (Verteilungszahlen, Distributive o Plurative) español cada se forma con je o jeweils, mientras que sendos también se formula con jeweils. Hallamos je26 veces y jeweils ocho veces en el subcorpus alemán. Cada, cada uno y sus equivalentes jede, jeder, jedes, jedem y jeden aparecen asombrosamente con la misma frecuencia. Observamos que, a pesar de que estos cuantificadores se emplean tanto en el subcorpus alemán como en el español de manera habitual, existen diferencias de frecuencia, por ejemplo en el cuantificador universal dual beide y ambos. Beide aparece con una destacada frecuencia de 70 apariciones mientras que ambos/ ambas aparece 37 veces lo que supone una gran diferencia a la baja en comparación con el alemán. El subcorpus español, no obstante, compensa esta diferencia empleando 24 veces los dos y 19 las dos. La expresión ambas/os dos, que en sí es una redundancia, ha sido incluida en el diccionario de la RAE, pero no surge en CrisCorp. Tampoco hallamos alle beide que existe en alemán, pero no tiene la connotación agramatical del español. Podemos concluir que en español se utilizan más expresiones de cantidad sinonímicas que en alemán, donde beide es la manera habitual de referirse a dos personas o cosas.

Otro tema de interés evidente es el grado de exactitud con el que se exponen los datos en los textos económicos. Los números partitivos formados a partir de números ordinales

(9) A pesar de que se trata de una forma anticuada, en algunos textos jurídicos alemanes aún se puede encontrar el sustantivo Zweitel.

(10) Etimológicamente la terminación en -tel proviene de la palabra Teil (parte).

(11) A excepción de doppelt (doble), no obstante, de la misma manera se puede decir zweifach.

(12) Entre paréntesis se presenta la cantidad de veces que el término de búsqueda aparece en el corpus.

(13) Se trata de un pronombre coloquial que funciona como un número inexacto con un alto valor que puede usarse también en forma de compuesto: zigtausend, zigmal, zigfach. 
pertenecen a la partición imprecisa si estos ordinales, que generalmente indican cantidades exactas, van acompañados de determinados adverbios como apenas, más de, cerca de o en alemán de mehr als, fast o etwa.

En cuanto al uso coinciden las dos lenguas y no se detectan grandes diferencias, más que de preferencia por una expresión u otra como, por ejemplo, zwischen y entre. El español emplea este cuantificador cuatro veces más que el alemán, mientras que en lengua alemana existe una clara predilección por rund, que, comparativamente, constituye más de la mitad del total de cuantificadores aproximativos en español. Las alternativas a rund (134) son ungefähr (2), mehr oder weniger (2), praktisch (6), etwa (84) y gut (30). Aparte de entre (60), que implica un lugar entre dos valores y es el cuantificador vago más utilizado en el subcorpus español, destacan otros como casi (48), cerca de (36), apenas y escaso (14). Estos últimos hacen referencia a valores por debajo del umbral de lo suficiente.

\section{Conclusiones}

Hemos intentado aunar en este trabajo la teoría de ambas lenguas y aplicarla a la práctica mediante un análisis con corpus. Estamos conscientes de que ambas partes podrían haberse intensificado dando lugar a un trabajo más largo y detallado. Sin embargo, nuestra intención era ofrecer una panorámica descriptiva de las referencias bibliográficas más importantes de la cuantificación para el español y el alemán, por un lado; y aplicar los resultados de dichos estudios en un corpus comparable, por otro (véase Stender 2016). De la primera parte, las conclusiones más relevantes son: 1) los estudios para ambas lenguas coinciden en la dificultad de atribuir categorías gramaticales a la cuantificación, pues algunos pueden ser tanto precisos como partitivos, por mencionar un ejemplo, además en muchos casos se trata de interpretaciones semánticas de lo que uno considera mucho o poco; 2) los tipos de cuantificadores e incluso las denominaciones son muy variados según los autores consultados y es difícil aunar las diferentes clases de palabras debajo de un solo paraguas; 3 ) además de los numerales y adverbios de cantidad, los adjetivos, sustantivos y verbos que denotan cantidad deben ser incluidos en los estudios sobre la cuantificación; 4) algunas clases de cuantificadores, pueden suponer una fuente de dificultades para la traducción ya que en una y en otra lengua no se emplean con la misma frecuencia (ambos, beide) o incluso no existen equivalentes directos (i.e. quincena, decena). En lo referente a las conclusiones sobre el contraste, las lenguas española y alemana no cuantifican de forma muy diferente, si bien algunas diferencias son: el alemán usa mayor número de cuantificadores vagos (knapp, etwa), existen diferencias en el uso de abreviaturas o de cuantificadores temporales (vierzehn Tagefrente a quincena, Quartalfrente a trimestre). Tampoco se escribe en español el término mil millones desarrollado, sino siempre se emplean los números seguido del sustantivo millones (1000 millones). Podemos afirmar, además, que la capacidad de composición alemana también ha alcanzado a los numerales, pues podemos ver ejemplos como Milliardenbeträge.

Bibliografia

Alarcos Llorach, E. (1999): Consideraciones sobre el neologismo. En El neologismo necesario. Fundación EFE, Madrid.

Altmann, Hans (1976): Die Gradpartikeln im Deutschen. Untersuchungen zu ihrer Syntax, Semantik und Pragmatik (Linguistische Arbeiten 33), De Gruyter, Tübingen.

Ballweg, J. (2003): Quantifikation und Nominaltypen im Deutschen, Gunter Narr Verlag, Tübingen.
Brauße, Ursula (2000): „Die Partikel allein. Klassifizierungs- und Bedeutungsprobleme", en Weydt, H. y Poljakova, S. (eds.) Linguistik Online, [Online] vol. 6, número 2. [https:// bop.unibe.ch/linguistik-online/article/ view/1008/1665].

Bußmann, H. (2002): Lexikon der Sprachwirtschaft. Kröner: Stuttgart.

Castell Vicente, A. (2002): Gramática de la lengua alemana. Múnich: Hueber Verlag.

Delbecque, N., Verveckken, K. (2012): Conceptually driven analogy in the grammaticalization of Spanish binominal quantifiers. En Linguistics 52 (3), 637-684

Eschenbach, C. (2013): ZählangabenMassangaben, Bedeutung und konzeptuelle Interpretation von Numeralia. Wiesbaden, Springer Fachmedien.

Gottschall, C. (2008): Prädikatenlogik der ersten Stufe mit Identität. En Skriptum zur Vorlesung Einführung in die Logik von Dr. Klaus Dethloff. [Online] [Documento de Internet disponible en http://logik.phl.univie.ac.at/ chris/ skriptum/skriptum.pdf]. 
Gutiérrez Rodríguez, E. (2008): Rasgos gramaticales de los cuantificadores débiles. Tesis doctoral dirigida por Ignacio Bosque. Facultad de Filología, Universidad Complutense de Madrid.

Gvozdanović,J.(2006): “Quantifizierende Adverbien und Typologie des Aspekts: Zur Mehrdimensionalität temporaler Kategorien", en Breindl, E., Gunke, L. y Strecker, B. (eds.), Grammatische Untersuchungen, Analysen und Reflexionen. Festschrift für Gisela Zifonun, Narr, Tübingen, 319-338.

Hirt, H. (1927): Indogermanische Grammatik. Teil III. Das Nomen. Indogermanische Bibliothek. Erste Abteilung: Sammlung indogermanischer Lehr- und Handbücher. 1. Reihe Grammatiken 13. Winter, Heidelberg.

Hentschel, E. y Weydt, H. (2003): Handbuch der deutschen Grammatik. De Gruyter: Berlín, Boston.

Hentschel, E. y Weydt, H. (1989): Wortartenprobleme bei Partikeln, en Weydt (ed.) Sprechen mit Partikeln, De Gruyter, Berlín, Boston, 3-19.

Kirchner,A.R.(2000): Einekopfgesteuerte Phrasenstrukturgrammatik für spanische Nominalphasen. Universidad de Göttingen, Tesis doctoral.

König, E. (1991): «Gradpartikeln», en Steger, H.y Wiegand,H. E. (eds.), Semantik/Semantics. Ein internationales Handbuch der zeitgenössischen Forschung, De Gruyter, Berlín, Boston, 786-803.

Labrador de la Cruz, M.B. (2005): Estudio contrastivo de la cuantificación inglés-español. León: Universidad de León.

Lamíquiz Ibáñez, V. (1991): La cuantificación lingüística y los cuantificadores UNED, Madrid.

Leonetti, M. (2007) Los cuantificadores. Arco Libros, Madrid.

Löbner, S. (1985): Natürlichsprachliche Quantoren: Zur Verallgemeinerung des Begriffs der Quantifikation, en Studium Linguistik 17/18, 79-113.
Martínez García, Hortensia (2007): Estructura y cuantificación partitiva. [Online] [Documento de Internet disponible en http://dialnet.unirioja.es/ servlet/articulo? codigo $=2898344]$.

Medina Reguera, A. y Stender, A. (2013): Quantifiers in a Spanish and German comparable corpus: a contrastive study based on specialized economic written on-line media texts. En Corpus Resources for Descriptive and Applied Studies. Current Challenges and Future Directions: Selected Papers from the 5th International Conference on Corpus Linguistics (CILC2013). Elsevier, Procedia - Social and Behavioral Sciences 95, 372-381,

Medina Reguera, A., Stender, A. (2012): El lenguaje económico español y alemán a través de la prensa especializada. Parámetros para un estudio contrastivo de corpus. En: Martino Alba, P., Lebsanft, C. (eds.): Telar de traducción especializada. Editorial Dykinson, Madrid, 79-89.

Meilán García, A. (1998): Construcciones locativas y cuantitativas. Arco, Madrid.

Pérez Berenguel, J.F. (2003): Glosario de errores comunes en la traducción económica y financiera. En Muñoz Martín, Ricardo (ed.) I AIETI. Actas del I Congreso Internacional de la Asociación Ibérica de Estudios de Traducción e Interpretación. Granada: AIETI 2, 619-628. [Online] [Documento de Internet disponible en: http://www.aieti.eu/pubs/actas/I/ AIETI_1_JFPB_Glosario.pdf].

Sánchez López, Cristina (1999): Los cuantificadores, capítulo 16. En Bosque I. y Demonte V. (eds.) Nueva Gramática Descriptiva de la Lengua Española. Espasa-Calpe, Madrid, 1025-1128.

Stender, Alice (2016): El lenguaje económico alemán y español de la prensa especializada: análisis basado en un corpus de la crisis económica (CRISCORP). Tesis Doctoral. Universidad Pablo de Olavide.

Vater, H. (1996): „Determinantien, Pronomina, Quantoren“, en Pérennec, M.-H. (ed.), Proformen des Deutschen,
Stauffenburg, Tübingen, 191-209.

Vater, H. (1984): „Determinantien und Quantoren im Deutschen“, en Zeitschrift für Sprachwissenschaft 3, 19-42.

Vervecken, K. (2015): Binominal Quantifiers in Spanish: Conceptuallydriven Analogy in Diachrony and Synchrony. De Gruyter, Berlín.

Wang, A., Piao, S. (2007): Translating Vagueness? A Study on Translations of Vague Quantifiers in an EnglishChinese Parallel Corpus. En Proceedings of the Corpus Linguistics Conference 2007, Birmingham, UK.

Weinrich, H. $\quad\left(2005, \quad 3^{\mathrm{a}} \quad\right.$ ed.): Textgrammatik der deutschen Sprache. Georg Olms Verlag, Hildesheim, Zúrich, Nueva York.

Wiese, H. (2004): Sprachvermögen und Zahlbegriff. En Schneider, Pablo \& Wedell, Moritz (eds.), Grenzfälle von Bild, Schrift und Zahl. Weimar: VDG Visual Intelligence Series 6, 123-145.

Wiese, H. (2001): Was unterscheidet Numeralia von anderen Ausdrücken? En (información no disponible). [Online] [Documento de Internet disponible en: http://publikationen. ub.uni- frankfurt.de/files/14108/ HWZahlw].

Wiese, H. (1996a); Der Status von Numeralia: Ein Beitrag zur Klärung des Klassifikationsproblems für Kardinalia, Ordinalia und NummerKonstruktionen. [Online] [Documento de Internet disponible en: https://www. academia.edu/6221111/Der Status von_Numeralia._Ein_Beitrag_zur_ $\mathrm{Kl} \% \mathrm{C} 3 \% \mathrm{~A} 4$ rung_des_

Klassifikationsproblems_f\%C3\%BCr Kardinalia Ordinalia und NummerKonstruktionen].

Wiese, H. (1996b): Zahl und Numerale. Eine Untersuchung zur Korrelation konzeptueller und sprachlicher Konstruktionen. Tesis doctoral. Humboldt-Universität Berlín.

Zamparelli, R. (2000): Layers in the Determiner Phrase. Garland, Nueva York y Londres. 